\title{
Research on the Relationship between Community Opening and Road Traffic Based on Traffic Flow
}

\author{
Yuhao Li \\ Department of Electrical Engineering, North China Electric Power University, Baoding, China \\ email:941582424@qq.com
}

\begin{abstract}
Keywords: Analytic Hierarchy Process, Community Opening, Traffic Flow Theory, Traffic Probability Distribution, Vissim Traffic Simulation System
\end{abstract}

\begin{abstract}
Firstly, making the equivalent problem and transform the problem into "The evaluation of the surrounding roads in the district to increase the intersection and a certain way after the connection, the surrounding junctions and road sections of the impact of the situation." And then we constructs a comprehensive evaluation index system by analytic hierarchy process. Secondly, based on the existing data related to vehicle traffic and the theory of probability and statistics, we established a mathematical model of vehicle traffic. Finally, we use the vissim traffic simulation system to simulate the mathematical model and the comprehensive evaluation index system.
\end{abstract}

\section{Introduction}

The State Council issued "on the further strengthening of urban planning and construction management of a number of opinions" in February 21, 2016 , of which Article XVI on the promotion of street system, in principle, no longer building a closed residential area, built residential quarters and units The hospital to gradually open and other views, attracted wide attention and discussion. In addition to issues such as security that may lead to opening an area, one of the focuses is whether open-air communities can optimize the road network structure, improve road capacity, improve traffic conditions, and improve the effectiveness. One view that closed-cell destruction of the urban road network structure, blocking the city "capillaries", likely to cause traffic congestion. After the opening of the district, the road network density increases, the road area increases, the traffic capacity will naturally improve. Some people think that this area with the district, location, external and internal road conditions and many other factors, can not be generalized. Some people think that after the opening of the district, although the road can be increased, accordingly, the district on the main road into and out of the intersection of the intersection of vehicles will increase, may also affect the main road traffic speed. On the open area on the impact of the surrounding road to study, to provide a quantitative basis for scientific decision-making.

\section{Analysis on the Influence of Community Opening}

Analysis of Urban Road Traffic. After the search data which can be found, the current traffic system (referring to the district has not yet opened), the degree of urban traffic congestion or smooth assessment of the main indicators are: saturation rate, space average speed, travel time, delay, queuing, parking[1].

Saturation: refers to the actual capacity of traffic facilities (roads, intersections, etc.) and traffic capacity ratio. The saturation rate reflects the busyness of the transport facility. It can be simply assumed that the closer the saturation rate is to 1, the closer the traffic facility is to saturation and the more busy the road. If the saturation rate is greater than 1 , the transport facility is supersaturated, which means that the road is very congested.

Spatial average speed: refers to the given time, a section of all vehicles on the average time travel distance. In general, the faster the average space, indicating the vehicle in the road congestion time, parking waiting time and the number of less, the better the road conditions. 
Travel time: the time it takes for a vehicle to travel for a while. The longer the travel time, the longer the vehicle will consume on the road and the worse the road condition from one side.

Delay: refers to the actual travel time and the ideal travel time difference. Delay is easy to make the driver fatigue and increase the loss of all aspects of the vehicle, such as fuel increases, vehicle wear and tear, etc., is not expected to happen.

Number of stops: the number of stops in the normal traffic of the vehicle and not yet reached the destination, indicating the road congestion. The more the number of stops, the more the number of hindered vehicles, the worse the road conditions.

Analysis of Urban Road Access after Community Opening. Opening community will have an impact on the district's internal road structure and the surrounding roads. In the interior of the district, the open road is changed from the internal road to the public road, the road area and network density will be increased; For the surrounding roads, the main road intersection around the original area will be increased. This problem can be equivalent to: along with additional traffic lights increasing will increase the trunk road vehicles through the intersection of waiting time, affecting the main road vehicle speed.

The Evaluation Index Of The Surrounding Roads After The Opening Of The Community. According to the evaluation of urban road access indicators, combined with the discussion caused by the community opening and the use of analytic hierarchy process theory, the district open to the impact of the surrounding roads into three layers: the target layer: the opening of the surrounding roads on the impact; Decision Layer: road network capacity, the main road speed, the district factor (community factors include the road traffic within the district, traffic flow, etc.); program layer: road capacity, the main road speed, cross-link integrated service level index. Because of the different geographical location of the four sides of the building in different circumstances, in the establishment of evaluation index system in the process, we only open one of the area of the above indicators for analysis.

Step 1: Road Traffic Capacity. The road capacity is the maximum traffic volume that can be used per unit time only under certain traffic conditions, road conditions and recognition criteria. The road capacity can be quantified by road capacity. There are many ways to divide the road capacity. In order to facilitate the analysis of the problem, the basic traffic capacity[1] reflects the general situation and simplifies the problem. The road traffic capacity is calculated as follows:

$$
C B=1000 \frac{v}{l_{0}}
$$

$v$ indicates the speed of travel and the unit is $\mathrm{km} / \mathrm{h}, l_{0}$ indicates the minimum distance of the auto's front and the unit is m.Overall, the greater the value of the capacity, indicating that the more vehicles can be accommodated on the road; the other hand, the number of road vehicles in a certain situation, the vehicle will be more smooth and effectively alleviate the road congestion situation.

Step 2: The Speed Of Autos On Main Roads. As the opening of the district to increase the intersection of traffic lights will increase the intersection of vehicles, thus affecting the vehicle speed on the main road of the surrounding area, so select the main road speed on the main road for analysis. According to the definition of speed, the speed is determined by the distance and time. On the side of the trunk road in terms of the car through the distance that is its long road, is the fixed value. But because the district is open, the main road to increase the intersection, the vehicle through the intersection need to stop waiting, the time calculation will change.

Assuming that the area is open, the main road is divided into $(n+1)$ segments as shown in the following figure 1 . 


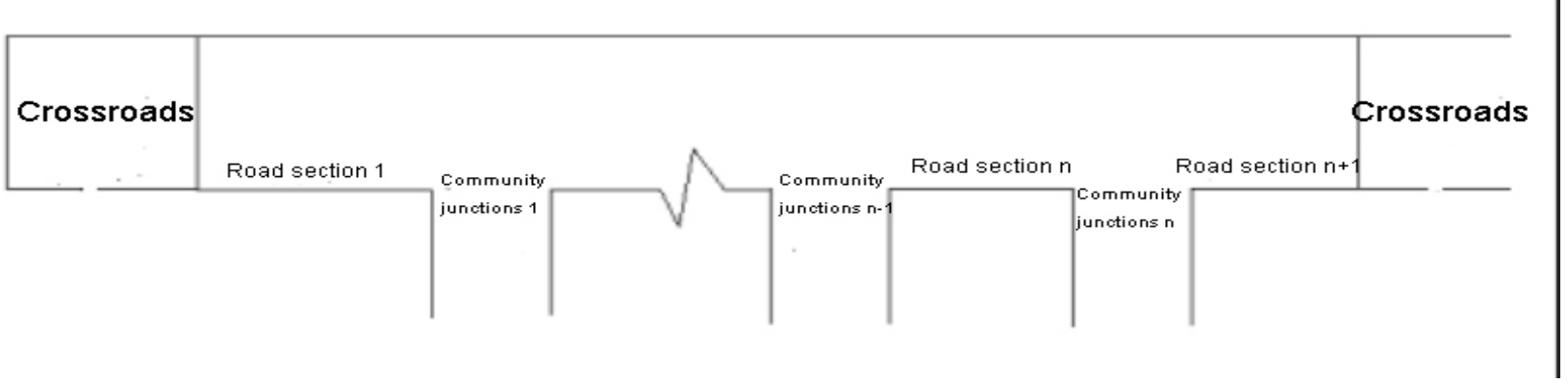

Fig.1.Community road structure

With $i$ mean road number, $\mathrm{j}$ mean the vehicle number, then for the $i$-th section, the completion of

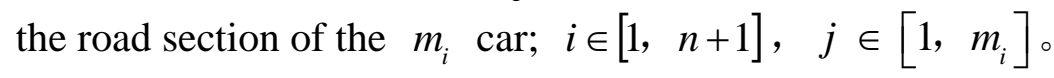

With $T_{i j}$ that the travel time of the $j$-th car of the $i$-th section , there are:

$$
T_{i}=\frac{1}{m_{i}} \sum_{j=1}^{m_{i}} T_{i j}
$$

Then the total travel time of a car on this road can be expressed as:

$$
T=\sum_{i=1}^{n+1} T_{i}
$$

Accordingly, the main road speed expression is:

$$
v=\frac{l}{T}
$$

The greater the speed of the main road, indicating that the shorter the travel time of the vehicle on the road, the traveler will be able to reach the destination more quickly, not only reduces the time spent by the traveler on the road, but also gives the traveler more comfortable and pleasant Feelings.

Step3:Community Factor---Intersection Integrated Service Level Index. In view of the increase in the main road after the opening of the district is the intersection of the increase, taking into account the intersection caused by the delay, queuing and parking times, the definition of the intersection of integrated service level index[2], to reflect the overall traveler Traffic conditions. It is reasonable and necessary to measure the impact of the district factor on the impact of the surrounding roads by selecting the service level index at the intersection. The cross-link integrated service level index is determined by the number of vehicles waiting cycles [2]. The idea is as follows: The total number of vehicles passing through the parking line for each semaphore cycle is $\mathrm{N}$. In practice, the vehicle waiting time is generally not the number of integer cycles, so the range to indicate that the number of vehicles in various traffic conditions are as follows:

$\begin{cases}n_{0}, & \text { The number of vehicles passing directly through the stop line } \\ n_{1}, & \text { The number of vehicles waiting for the cycle }<=1 \text { times before stopping the line } \\ n_{2}, & \text { The number of vehicles waiting for the cycle belong }(1,2] \text { times } \\ n_{3}, & \text { The number of vehicles waiting for the cycle belong }(2,3] \text { times } \\ n_{4}, & \text { The number of vehicles waiting for the cycle belong }(3,4] \text { times }\end{cases}$

tips: The number of waiting cycles is simply equivalent to the number of signal periods

$p_{0}, p_{1}, p_{2}, p_{3}, p_{4}$ respectively represent the percentage of vehicles passing through the total number of vehicles. In the case of vehicle traffic, $p_{0}+p_{1}$ close to $100 \%$; when the traffic situation deteriorated, congestion occurs, $p_{2}, p_{3}, p_{4}$ value began to increase, when $p_{4}$ close to $100 \%$ when the traffic situation is very bad, serious vehicle congestion, basic can not pass normally. In practice, the waiting cycle of the vehicle is not easy to use, with the recursive formula can be intuitively identified: 


$$
\begin{aligned}
& L_{1}(k)=\max \{0, L(k)-N(k)\} \\
& L_{2}(k)=\max \left\{0, L_{1}(k-1)-N(k)\right\} \\
& L_{3}(k)=\max \left\{0, L_{2}(k-1)-N(k)\right\} \\
& n_{4}(k)=\min \left\{L_{3}(k-1), N(k)\right\} \\
& n_{3}(k)=\min \left\{L_{2}(k-1)-L_{3}(k-1), N(k)-n_{4}(k)\right\} \\
& n_{2}(k)=\min \left\{L_{1}(k-1)-L_{2}(k-1), N(k)-n_{4}(k)-n_{3}(k)\right\} \\
& n_{1}(k)=\min \left\{L(k-1)-L_{1}(k-1), N(k)-n_{4}(k)-n_{3}(k)-n_{2}(k)\right\} \\
& n_{0}(k)=N(k)-n_{4}(k)-n_{3}(k)-n_{2}(k)-n_{1}(k)
\end{aligned}
$$

among them:

$n_{0,1,2,3,4}(k)$. The number of vehicles waiting for 0-4 cycles in the vehicle through the parking line within the $k$-th cycle; $N(k)$ :Number of vehicles passing through the stop line within the $k$-th cycle; $L(k)$ :the length of the vehicle queue which is not leaving within the $k$-th cycle; $L_{1}(k)$ :Number of vehicles not leaving within the $k$-th cycle; $L_{2}(k)$ : the number of vehicles waiting to be greater than or equal to 2 in the unmounted vehicle within the k-th cycle; $L_{3}(k)$. The number of vehicles waiting to be greater than or equal to 3 in the unmounted vehicle within the $k$-th cycle.

This defines the cross-link integrated service level index as follows:

$$
\text { LOSI }=\left(1-\frac{a_{0} p_{0}+a_{1} p_{1}+a_{2} p_{2}+a_{3} p_{3}+a_{4} p_{4}}{a_{4} \times 100 \%}\right) \times 100
$$

$a_{0} \ldots a_{4}$ represents the weighting factor, $a_{0}=0, a_{1}=1, a_{2}=3, a_{3}=6, a_{4}=10$.

When the service level index is 100 , indicating that the vehicle through the intersection are smooth, no parking waiting, is the ideal traffic conditions; when the service level index is greater than 90, indicating that all vehicles through the intersection of up to wait for a cycle, which belongs to normal Of the traffic conditions; when the service level index is 0 , indicating that all traffic through the intersection of vehicles waiting for more than or equal to four, which is the worst traffic conditions.

Final step. In order to facilitate the comprehensive trade-off of the above three factors, the establishment of community after the opening of the surrounding roads on the pros and cons of the evaluation system as follows:

$$
Y=\alpha \frac{C B^{\prime}-C B}{C B}+\beta \frac{v^{\prime}-v}{v}+\gamma \frac{L O S I^{\prime}-L O S I}{L O S I}
$$

$\alpha, \beta, \gamma$ are the traffic capacity, the main road speed, the intersection of the comprehensive service level index weight. In view of the opening of the district on the impact of the surrounding road capacity of the largest, may consider taking $\alpha=0.5$; main road speed is an important indicator of whether the road is smooth, take $\beta=0.3$; then the intersection of the comprehensive service level index of the proportion of $\lambda=0.2 . C B, v, L O S I$ said the district before the opening of the situation, $C B^{\prime}, v^{\prime}, L O S I^{\prime}$ said the district after the opening of the corresponding statistical data.

The principle of road traffic assessment is that if the value is greater than 0 , it can be considered that the comprehensive balance of all factors, the district open to the surrounding road traffic is beneficial, and the larger the value, indicating that the opening of the surrounding roads more beneficial to the surrounding roads; The value is less than 0 , then the opening of the district is not conducive to road conditions improved.

\section{Mathematical model of vehicle traffic}

Step 1: Mathematical model of vehicle speed. Taking into account the small car body length is generally $3-5 \mathrm{~m}$, set the minimum body length of $3 \mathrm{~m}$, the maximum body length of $7 \mathrm{~m}$, take the car from the distance $1 \mathrm{~m}$ for the vehicle can pass the minimum distance, to determine the minimum 
head spacing threshold of $6 \mathrm{~m}$ [3], the largest head spacing For $30 \mathrm{~m}$. If the measured data of the head spacing is greater than $30 \mathrm{~m}$, then the vehicle is considered the ideal free driving state; if the head spacing is less than $6 \mathrm{~m}$, indicating that the road is in a serious obstruction state, the vehicle stops moving.

The statistics[3] show that when the head spacing is [6,9), the negative exponential fitting error is less than 0.001 , and the vehicle speed follows the negative exponential distribution under the high density traffic flow. When the head spacing is $[9,30]$, the velocity follows the logarithm State distribution [3].According to the speed of the fitting function distribution map can be drawn:

The average speed of the vehicle is approximately $2 \mathrm{~m} / \mathrm{s}$ when the head spacing is [6,9].

When the head spacing is [9,30], the average speed of the vehicle is approximately $8.3 \mathrm{~m} / \mathrm{s}$.

Step 2: The mathematical model of vehicle direction. Taking into account the complexity of the district and the surrounding roads, the intersection may have crossroads, T-junctions, oblique cross-border intersection, Y-shaped intersection and other different circumstances, in order to facilitate the analysis of the problem, assuming that the intersection near the district are crossroads. According to experience, the intersection of traffic distribution is: turn $40 \%, 40 \%$ straight, turn right $20 \%$.Community opening is equivalent to the original district in the surrounding roads on the alteration of an intersection, assuming that after the opening of the original lane will have $30 \%$ of the vehicles choose to enter the district road.

Different traffic flow conditions are different, in order to facilitate the vehicle to ease, keep the vehicle smooth, take the Webster timing method [4] for different lights phase were time, as follow the Table 1 and Table 2:

Table 1. peripheral road crossroads signal timing scheme

\begin{tabular}{|c|c|c|c|c|}
\hline & $\mathrm{A}$ & $\mathrm{B}$ & $\mathrm{C}$ & $\mathrm{D}$ \\
\hline $\begin{array}{c}\text { Phase } \\
\text { description }\end{array}$ & \multicolumn{1}{c}{$\mathrm{C}$} & & \\
\hline Green time & $47 \mathrm{~s}$ & $26 \mathrm{~s}$ & $47 \mathrm{~s}$ & $26 \mathrm{~s}$ \\
\hline
\end{tabular}

Signal cycle: 158s; Yellow light time: 3s;Green light interval: 3s.

Table 2. residential road junction signal junction scheme

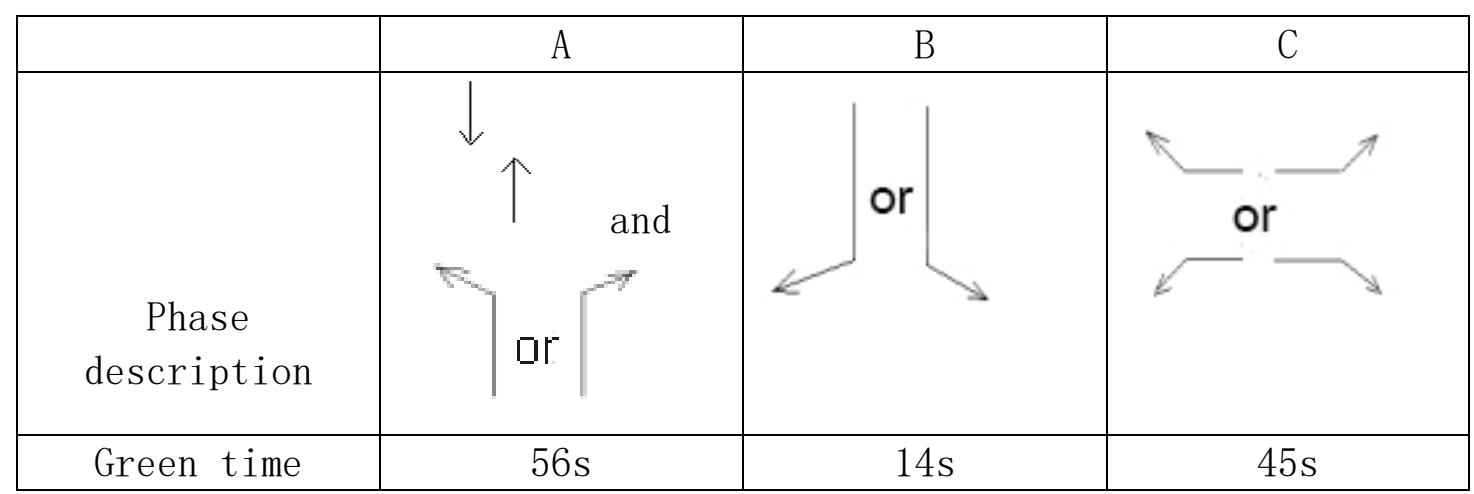

Signal cycle: 124s; Yellow light time: 3s;Green light interval: 3s.

Step 3 Traffic density and average speed. The relationship between road traffic volume and average speed, traffic density is as follows[5]:

$$
Q=\mu \cdot \bar{v} \text {. }
$$

With $\mu^{\prime}, Q^{\prime}, \bar{v}^{\prime}$, respectively, after the change in traffic density, traffic and average speed, set the 
traffic density change rate of $a$, the speed of change is:

$$
\overline{v^{\prime}}=\left(1-\frac{a}{1+a}\right) \bar{v}
$$

For the different types of cells, the district after the opening of the internal structure of the road is different, the distribution of the main road traffic will also have an impact on the integration of the vehicle speed and direction of the analysis, the surrounding traffic density will change the use of the vehicle speed and direction of the model will be able to comprehensively study the impact of vehicle traffic on road traffic.

\section{Test Results}

We designed two different communities, the results shown in Figure2 (a) and (b):

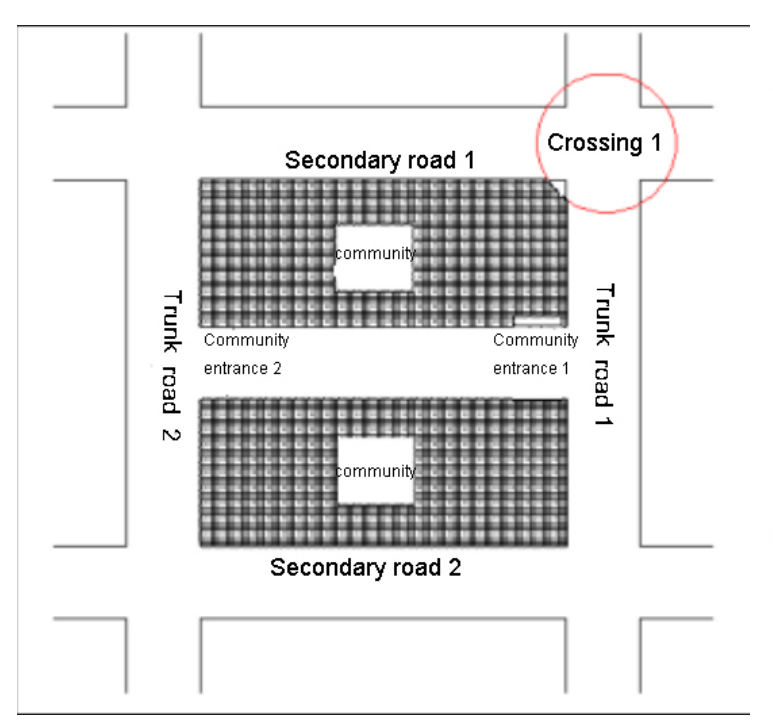

(a) Type I

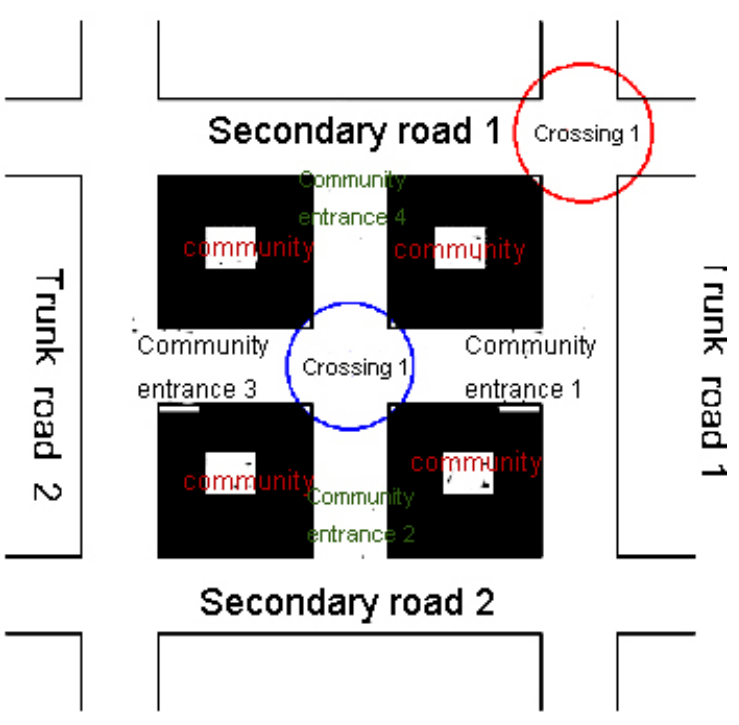

(b) Type II

Fig.2.Community type

Using the above two models to analyze them, get the following results which shown in Table 3:

Table 3. Evaluation index score

\begin{tabular}{|c|c|c|c|c|c|}
\hline \multirow{2}{*}{ Community number } & & $C B$ & $v$ & LOSI & $Y$ \\
\hline \multirow{2}{*}{ Type I } & Before the district is open & 415 & 8.3 & 92 & \multirow{2}{*}{-0.176} \\
\cline { 2 - 6 } & After the district is open & 415 & 3.443 & 92 & \\
\hline Community number & & $C B$ & $v$ & LOSI & $Y$ \\
\hline \multirow{2}{*}{ Type II } & Before the district is open & 415 & 8.3 & 92 & \multirow{2}{*}{0.155} \\
\cline { 2 - 6 } & After the district is open & 415 & 3.49 & 92.5 & \\
\hline
\end{tabular}

In the case of community type II, after the opening of the district, the traffic speed of the main road decreased due to the addition of the intersection, but the traffic density of the main road was reduced due to the opening of the road, and the traffic level of the intersection was increased. Increased traffic capacity. Compared to the type of residential one, due to the comprehensive score 
becomes larger, the opening of the two types of traffic on the traffic situation has eased.

The traffic conditions before and after the opening of each type of community were simulated by the Vissim traffic simulation system and evaluated by another existing urban road traffic condition evaluation index (mean speed VT [2]). The results are as Figure 3:

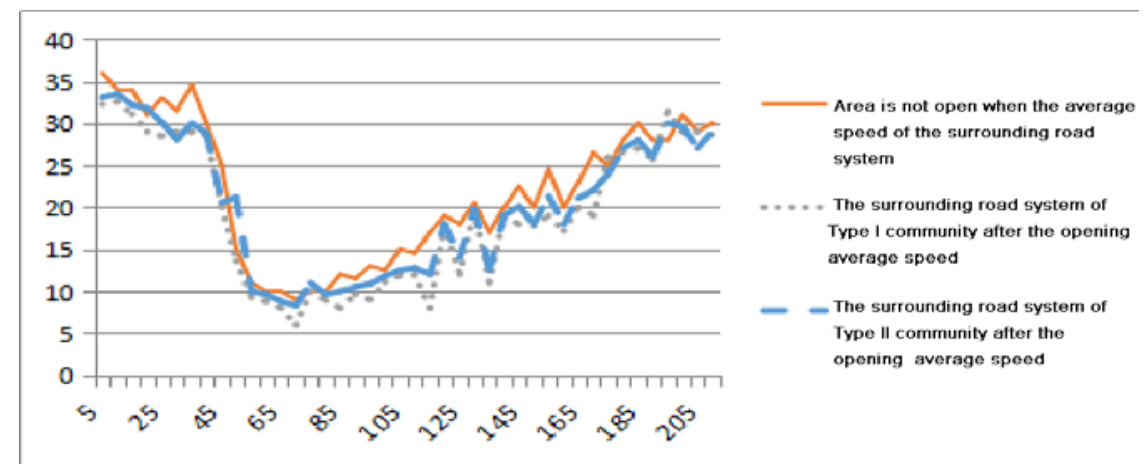

Fig.3.Simulation results

Corresponding to the road comprehensive evaluation index value $Y_{1}, Y_{2}$, each district before and after the opening of the average speed of the road network system changes $\Delta V T_{1}, \Delta V T_{2}$, to reflect the open area on the surrounding road traffic impact.

From the above chart we can see $V T_{1} \prec V T_{2} \prec 0$, and we established the $Y$ value of the evaluation results consistent, we proved the model and evaluation of the evaluation index system is reasonable and feasible.

\section{Conclusion}

In the current road conditions, the evaluation of road traffic conditions are saturated, spatial average speed, travel time, delay, queuing, parking number six. Based on the equivalent of community opening - "in the original area on the road around the intersection of additional roads", with the analytic hierarchy process to extract the road capacity, the main road speed, cross - level service level index of road conditions comprehensive evaluation index system. And based on the probability model of vehicle traffic, to build different types of cell discovery: the trunk road and secondary trunk road type is more suitable for open, and open the district should be selected the original traffic congestion, traffic flow larger position. Therefore, it is recommended that the urban planning department should give priority to traffic congestion when selecting the target open area, and open the way as far as possible to connect the main road and the secondary road. At the same time, in the new open area can be considered when the establishment of the district outside the border forkless section of the large area of the district to improve the main road speed.

\section{References}

[1]Ge Song. Calculation of road capacity. Exploration and Research.[2010-9-2].

[2]Evaluation of Urban Road Traffic Evaluation Method and Evaluation System. $<\underline{\text { http://www.docin.com/[2012-4-28] }>\text {. }}$

[3]Qi Wang, Li Li, Jianming Sun, Bin Zhou. Space Distribution of Traffic Flows at Different Head Spacing. Journal of Tsinghua University.2011.51(3).

[4]Webster Timing Method. <http://wenku.baidu.com/view/9f365615fad6195f312ba6cc.html>.

[5]Ming Chen. Study on Practical Relationship Model of Urban Road Traffic Speed. Southeast University.2004. 